\title{
Density Scaling for Multiplets
}

\author{
Á. Nagy \\ Department of Theoretical Physics, University of Debrecen, H-4010 Debrecen, Hungary
}

(November 16, 2010)

\begin{abstract}
Generalized Kohn-Sham equations are presented for lowest-lying multiplets. The way of treating non-integer particle numbers is coupled with an earlier method of the author. The fundamental quantity of the theory is the subspace density. The Kohn-Sham equations are similar to the conventional Kohn-Sham equations. The difference is that the subspace density is used instead of the density and the Kohn-Sham potential is different for different subspaces. The exchange-correlation functional is studied using density scaling. It is shown that there exists a value of the scaling factor $\zeta$ for which the correlation energy disappears. Generalized OPM and KLI methods incorporating correlation are presented. The $\zeta$ KLI method as simple as the original KLI method is proposed for multiplets .
\end{abstract}

\section{INTRODUCTION}

Density functional theory in the original form [1] is valid for the lowest-energy state in each symmetry class. The symmetry problem in the density functional theory has been attracted special attention. [2-5]. The first methods to treat the multiplet structure developed by Bagus and Bennett [6], Ziegler, Rauk and Baerends [7], von Barth [8] and Wood [9], have the feature of not being completely within the frame of the density functional theory. The method of fractionally occupied states of Gross, Oliveira and Kohn [10] has been 
used to treat the multiplet problem [11] and the exchange potential has been determined for ensembles of low-lying multiplets. The present author generalized a method of subspaces by Theophilou [12] to treat multiplets $[13,14]$. Now this method is extended and combined with density scaling $[16,17]$.

Density scaling was proposed by Chan and Handy [15]. In density scaling the density $n(\mathbf{r})$ is changed to $\zeta n(\mathbf{r})$. It is shown that there exists a value of the scaling factor for which the correlation energy disappears. The optimized potential method (OPM) [18] and the Krieger-Li-Iafrate (KLI) [19] approach are generalized to incorporate correlation.

In section 2 the subspace theory for multiplets is reviewed. Section 3 presents generalized Kohn-Sham equations for multiplets based on density scaling. In section 5 the $\zeta \mathrm{OPM}$ and $\zeta$ KLI methods are detailed for atomic multiplets. In section 6 results for some atomic multiplets are presented and discussed. In the Appendix the density and the kinetic energy of spherically symmetric systems are derived.

\section{SUBSPACE THEORY FOR MULTIPLETS}

The constrained search is applied on "subspace density matrices" constructed from the wave functions of the given multiplet.

$$
\hat{D}^{\gamma}=\sum_{i=1}^{g^{\gamma}} \omega_{i}^{\gamma}\left|\Psi_{i}^{\gamma}\right\rangle\left\langle\Psi_{i}^{\gamma}\right| .
$$

This subspace is spanned by a set of wave functions of the given symmetry $\gamma$ : $\Psi_{i}^{\gamma}$. The dimension of the subspace is equal to the degeneracy $g^{\gamma}$. The weighting factors $\omega_{i}^{\gamma}$ should satisfy the conditions

$$
1=\sum_{i}^{g^{\gamma}} \omega_{i}^{\gamma}
$$

and

$$
\omega_{i}^{\gamma} \geq 0
$$

The energy of a multiplet with a given symmetry $\gamma$ can be given: 


$$
E^{\gamma}\left[n^{\gamma}\right]=\min _{\hat{D}^{\gamma} \rightarrow n^{\gamma}}\left[\hat{D}^{\gamma}\left(\hat{T}+\hat{V}_{e e}\right)\right]+\int n^{\gamma}(\mathbf{r}) v(\mathbf{r}) d \mathbf{r}
$$

where $\hat{T}$ and $\hat{V}_{e e}$ are, respectively, the kinetic and the electron-electron repulsion operators. $v$ is the external potential. In principle, any set of weighting factors $\omega_{i}^{\gamma}$ satisfying conditions (2) and (3) can be used. If the weighting factors $\omega_{i}^{\gamma}$ are all equal, the density has the property of transforming according to the totally symmetric irreducible representation. So, for instance, for atoms the density will be spherically symmetric. In this case the subspace density

$$
n^{\gamma}=\sum_{i=1}^{g^{\gamma}} \omega_{i}^{\gamma} \int\left|\Psi_{i}^{\gamma}\right|^{2} d s_{1} d \mathbf{x}_{\mathbf{2}} \ldots d \mathbf{x}_{\mathbf{N}}
$$

has the symmetry of the external potential. ( $\mathbf{x}$ stands for both the coordinates and the spin.) Definining the functional

$$
F^{\gamma}\left[n^{\gamma}\right]=\min _{\hat{D}^{\gamma} \rightarrow n^{\gamma}}\left[\hat{D}^{\gamma}\left(\hat{T}+\hat{V}_{e e}\right)\right]
$$

the generalized Hohenberg-Kohn theorem has the form

$$
F^{\gamma}\left[n^{\gamma}\right]+\int n^{\gamma}(\mathbf{r}) v(\mathbf{r}) d \mathbf{r} \geq E_{0}^{\gamma}
$$

There is an equality if and only if the trial density $n^{\gamma}$ is equal to the true density of the multiplet $\gamma$. (The variational principle is valid here because we are considering the lowestlying energy level of symmetry $\gamma$.)

\section{GENERALIZED KOHN-SHAM EQUATIONS FOR MULTIPLETS}

In the standard density functional theory the Kohn-Sham system is constructed as a noninteracting system that has the same density as the real interacting one $[22,23,25,24]$. The standard Kohn-Sham equations can be obtained by the Levy-Lieb constrained search $[20,21]$

$$
T_{s}[n]=\min _{\Psi \rightarrow n}\langle\Psi|\hat{T}| \Psi\rangle .
$$


As a first step a Kohn-Sham scheme $[13,14]$ is defined. It is constructed by a constrained search over the density matrices $\hat{D}^{\gamma}$ that lead to density $n^{\gamma}$ :

$$
T_{s}\left[n^{\gamma}\right]=\min _{\hat{D}^{\gamma} \rightarrow n^{\gamma}} \operatorname{Tr}\left[\hat{D}^{\gamma} \hat{T}\right]
$$

The noninteracting density matrix is defined as

$$
\hat{D}_{s}^{\gamma}=\sum_{i=1}^{g^{\gamma}} \omega_{i}^{\gamma}\left|\Psi_{s, i}^{\gamma}\right\rangle\left\langle\Psi_{s, i}^{\gamma}\right|,
$$

where the noninteracting wave functions of symmetry $\gamma$ are constructed as a linear combination of Slater determinants $\Phi_{\kappa}$.

$$
\Psi_{s, i}^{\gamma}=\sum_{\kappa} c_{i \kappa}^{\gamma} \Phi_{\kappa} \quad\left(i=1,2, \ldots, g^{\gamma}\right)
$$

As a second step we generalize further the Kohn-Sham scheme $[16,17]$ and consider a non-interacting system with ground-state density $n_{\zeta}^{\gamma}(\mathbf{r})=n^{\gamma}(\mathbf{r}) / \zeta$, where $\zeta=N / N_{\zeta}$ is any positive number. If the original real system has $N$-electrons

$$
\int n^{\gamma}(\mathbf{r}) d \mathbf{r}=N
$$

the Kohn-Sham system has $N_{\zeta}$-electrons:

$$
\int n_{\zeta}^{\gamma}(\mathbf{r}) d \mathbf{r}=N_{\zeta}
$$

$N$ is always integer, but $N_{\zeta}$ is generally non-integer. The second generalized Kohn-Sham system is constructed by the constrained search over the density matrices $\Gamma^{\gamma}$ that lead to density $n_{\zeta}^{\gamma}$.

The functional $T_{\zeta}\left[n^{\gamma}\right]$ is defined as

$$
T_{\zeta}\left[n^{\gamma}\right]=\zeta \min _{\hat{\Gamma}^{\gamma} \rightarrow n_{\zeta}^{\gamma}} \operatorname{Tr}\left[\hat{\Gamma}^{\gamma} \hat{T}\right]
$$

The total noninteracting density matrix is defined as

$$
\hat{\Gamma}_{\zeta s}^{\gamma}=(1-q) \hat{D}_{s}^{\gamma}+q \hat{D}_{s}^{i o n}
$$

where 


$$
q=N-N_{\zeta}=N(1-1 / \zeta)
$$

We consider only the case where $q$ is a small positive number: $q<<1 . \hat{D}_{s}^{i o n}$ denotes the noninteracting ground-state density matrix of the ion that is obtained after ionization. The density corresponding to the density matrix $\hat{\Gamma}_{\zeta s}^{\gamma}$ is $n_{\zeta}^{\gamma}$ :

$$
n_{\zeta}^{\gamma}=(1-q) n^{\gamma}+q n^{i o n}
$$

$n^{i o n}$ is the density constructed from $\hat{D}_{s}^{i o n}$. Now, the generalized Kohn-Sham equations can be obtained by substituting Eq. (15) to Eq. (14) and carrying out the minimalization. Because of the fact that we have a linear combination of several Slater determinants the form of the Kohn-Sham equations might be rather complicated for an arbitrarily selected set of weighting factors and have to be derived separately for each desired case. For a spherically symmetric case and equal weighting factors, however, the Kohn-Sham equations have a very simple form as it is shown in the Appendix. In this case the noninteracting kinetic energy is given by

$$
T_{\zeta}^{\gamma}=\sum_{j=1}^{K} \lambda_{\zeta j} \int P_{\zeta j}^{\gamma}\left[-\frac{1}{2}\left(P_{\zeta j}^{\gamma}\right)^{\prime \prime}+\frac{l_{j}\left(l_{j}+1\right)}{2 r^{2}} P_{\zeta j}^{\gamma}\right] d r,
$$

where $P_{\zeta j}^{\gamma}$ and $\lambda_{\zeta j}$ are the radial wave functions and the occupation numbers corresponding to the given configuration, respectively. $K$ is the smallest integer for which $\lambda_{\zeta i}^{\gamma} \neq 0$. " denotes second derivative with respect to $r$. The radial density is

$$
\varrho_{\zeta}^{\gamma}=\sum_{j=1}^{K} \lambda_{\zeta j}\left(P_{\zeta j}^{\gamma}\right)^{2}
$$

The minimization of the noninteracting kinetic energy (18) keeping the density $n_{\zeta}^{\gamma}$ (Eq. (19)) fixed leads to the radial Kohn-Sham equations

$$
-\frac{1}{2}\left(P_{\zeta j}^{\gamma}\right)^{\prime \prime}+\frac{l_{j}\left(l_{j}+1\right)}{2 r^{2}} P_{\zeta j}^{\gamma}+v_{\zeta K S}^{\gamma} P_{\zeta j}^{\gamma}=\varepsilon_{\zeta j}^{\gamma} P_{\zeta j}^{\gamma},
$$

where

$$
v_{\zeta K S}^{\gamma}=v(\mathbf{r})+\int \frac{n^{\gamma}\left(\mathbf{r}^{\prime}\right)}{\left|\mathbf{r}-\mathbf{r}^{\prime}\right|} d \mathbf{r}+v_{\zeta x c}^{\gamma}\left(n_{\zeta}^{\gamma} ; \mathbf{r}\right),
$$


where $v_{\zeta x c}^{\gamma}$ is the exchange-correlation potential of the lowest lying multiplet of symmetry $\gamma$. An essential point in the theory is that the exchange-correlation potential and so the Kohn-Sham potential depends on $\gamma$, we have different potentials for different multiplets. (The fact that the exchange-correlation potential must in general be different for each symmetry multiplet was first pointed out by Weiner and Trickey [26].) The exchange-correlation potential depends also on the weighting factors.

In the original Kohn-Sham theory the exchange-correlation energy $E_{x c}[\varrho]$ is defined by the total energy expression

$$
E[n]=T_{s}[n]+J[n]+E_{x c}[n]+\int n(\mathbf{r}) v(\mathbf{r}) d \mathbf{r}
$$

where

$$
J[n]=\frac{1}{2} \int \frac{n\left(\mathbf{r}_{1}\right) n\left(\mathbf{r}_{2}\right)}{\left|\mathbf{r}_{1}-\mathbf{r}_{2}\right|} d \mathbf{r}_{1} d \mathbf{r}_{2}
$$

is the classical Coulomb energy. The functional derivatives of $J$ and $E_{x c}$ are $v_{J}$ and $v_{x c}$, the classical Coulomb potential and the exchange-correlaton potential, respectively. The Kohn-Sham potential is given by

$$
v_{K S}=v+v_{J}+v_{x c}
$$

where $v$ is the external potential. Now, the total energy of a given symmetry $\gamma$ can be written

$$
E\left[n^{\gamma}\right]=T_{s}\left[n^{\gamma}\right]+J\left[n^{\gamma}\right]+E_{x c}^{\gamma}\left[n^{\gamma}\right]+\int n^{\gamma}(\mathbf{r}) v(\mathbf{r}) d \mathbf{r}
$$

Similarly, in this modified Kohn-Sham theory the exchange-correlation energy $E_{\zeta x c}\left[n^{\gamma}\right]$ is defined by the total energy expression

$$
E^{\gamma}\left[n^{\gamma}\right]=T_{\zeta}\left[n^{\gamma}\right]+J\left[n^{\gamma}\right]+E_{\zeta x c}^{\gamma}\left[n^{\gamma}\right]+\int n^{\gamma}(\mathbf{r}) v(\mathbf{r}) d \mathbf{r}
$$

The modified Kohn-Sham potential has the form

$$
v_{\zeta K S}^{\gamma}=v+v_{J}^{\gamma}+v_{\zeta x c}^{\gamma} .
$$


A comparison of Eqs. (25) and (26) leads to the important relation

$$
T_{s}\left[n^{\gamma}\right]+E_{x c}^{\gamma}\left[n^{\gamma}\right]=T_{\zeta}\left[n^{\gamma}\right]+E_{\zeta x c}^{\gamma}\left[n^{\gamma}\right]
$$

Certainly, $T_{\zeta=1}=T_{s}$. Utilizing the constained search we obtain

$$
T_{\zeta}\left[n^{\gamma}\right]=\zeta T_{s}\left[n_{\zeta}^{\gamma}\right]
$$

The physical meaning of the density scaling can be seen from Eq. (28). A change in the non-interacting kinetic energy results a change in the exchange-correlation energy. In this paper the case when $\zeta$ is only slightly larger than 1 is studied. Then there is only a small change in the exchange-correlation energy. We search that $\zeta_{c}$ for which the correlation energy disappears: $E_{\zeta_{c}, c}[n]=0$.

Generalizing the correlation energy of the standard density functional theory, the correlation energy of the multiplet $\gamma$ is defined as

$$
E_{c}^{\gamma}\left[n^{\gamma}\right]=\operatorname{Tr}\left[\hat{D}_{n^{\gamma}}^{\min }\left(\hat{T}+\hat{V}_{e e}\right)\right]-\operatorname{Tr}\left[\hat{D}_{s n^{\gamma}}^{\min }\left(\hat{T}+\hat{V}_{e e}\right)\right]
$$

$\hat{D}_{n^{\gamma}}^{\min }$ denotes the density matrix that minimizes $\operatorname{Tr}\left[\hat{D}\left(\hat{T}+\hat{V}_{e e}\right)\right]$ and leads to the density $n^{\gamma} . \hat{D}_{s n^{\gamma}}^{\gamma}$ is the density matrix that minimizes the kinetic energy $\operatorname{Tr}[\hat{D} \hat{T}]$ and leads to the density $n^{\gamma}$.

Analogously, we define the modified correlation energy as

$$
E_{\zeta c}^{\gamma}\left[n^{\gamma}\right]=\operatorname{Tr}\left[\hat{\Gamma}_{n^{\gamma}}^{\gamma \min }\left(\hat{T}+\hat{V}_{e e}\right)\right]-\operatorname{Tr}\left[\hat{\Gamma}_{s n^{\gamma} \zeta}^{\gamma \min }\left(\zeta \hat{T}+\zeta^{2} \hat{V}_{e e}\right)\right]
$$

\section{Theorem}

There exists a parameter $\zeta^{\gamma}$ for which the correlation energy disappears: $E_{\zeta c}^{\gamma}=0$.

Proof:

If $\zeta=1, E_{\zeta c}^{\gamma}$ is equal to the density functional correlation energy $E_{c}^{\gamma}$, which is negative. Consider a small change in $\zeta$ and notice that from the definition (31) follows that $E_{\zeta c}^{\gamma}$ is almost quadratic in $\zeta \cdot E_{\zeta c}^{\gamma}=0$ means that

$$
\left.\zeta^{2} \operatorname{Tr}\left[\hat{\Gamma}_{s n \zeta}^{\gamma \min } \hat{V}_{e e}\right)\right]+\zeta \operatorname{Tr}\left[\hat{\Gamma}_{n}^{\gamma \min } \hat{T}\right]-\operatorname{Tr}\left[\hat{\Gamma}_{n}^{\gamma \min }\left(\hat{T}+\hat{V}_{e e}\right)\right]=0
$$


This equation has solutions as

$$
\left.\operatorname{Tr}\left[\hat{\Gamma}_{n}^{\gamma \min } \hat{T}\right]^{2}+4 \operatorname{Tr}\left[\hat{\Gamma}_{s n_{\zeta}}^{\gamma \min } \hat{V}_{e e}\right)\right] \operatorname{Tr}\left[\hat{\Gamma}_{n}^{\gamma \min }\left(\hat{T}+\hat{V}_{e e}\right)\right]>0
$$

Consequently, there exists a value of $\zeta^{\gamma}$ for which $E_{\zeta c}^{\gamma}=0$. Note that Eq. (32) has two solutions, however, the other solution is not close to 1 . Moreover it can even be negative and thus physically not acceptable.

\section{THE $\zeta$ OPM AND $\zeta$ KLI METHODS FOR ATOMIC MULTIPLETS}

The optimized potential method can be applied when the total energy $E^{\gamma}\left[u_{\zeta i}^{\gamma}\right]$ is given as a functional of the one-electron orbitals $u_{\zeta i}^{\gamma}$. The energy $E^{\gamma}\left[u_{\zeta i}^{\gamma}\right]$ is the lowest lying solution of symmetry $\gamma$ of the Hamiltonian of the system. The one-electron orbitals $u_{\zeta i}^{\gamma}$ are eigenfunctions of a local effective potential $V_{\zeta}^{\gamma}$

$$
\hat{h}_{\zeta}^{\gamma} u_{\zeta i}^{\gamma}=\left(-\frac{1}{2} \nabla^{2}+V_{\zeta}^{\Gamma}\right) u_{\zeta i}^{\Gamma}=\varepsilon_{\zeta i}^{\Gamma} u_{\zeta i}^{\Gamma}
$$

with $V_{\zeta}^{\gamma}$ being determined by requiring that $E^{\gamma}\left[u_{\zeta i}^{\gamma}\right]$ is minimized for all $u_{\zeta i}^{\gamma}$ obtained from Eq. (34). This results in

$$
\frac{\delta E^{\gamma}}{\delta V_{\zeta}^{\gamma}}=\sum_{i} \int \frac{\delta E^{\gamma}}{\delta u_{\zeta i}^{\gamma *}\left(\mathbf{r}^{\prime}\right)} \frac{\delta u_{\zeta i}^{\gamma *}\left(\mathbf{r}^{\prime}\right)}{\delta V_{\zeta}^{\gamma}(\mathbf{r})} d \mathbf{r}^{\prime}+\text { c.c. }=0 .
$$

The functional derivative of the one-electron orbitals $u_{\zeta i}^{\Gamma}$ with respect to the local effective potential $V_{\zeta}^{\gamma}$ can be calculated with the help of Green's functions:

$$
\begin{gathered}
\frac{\delta u_{\zeta i}^{\gamma *}\left(\mathbf{r}^{\prime}\right)}{\delta V_{\zeta}^{\gamma}(\mathbf{r})}=-G_{\zeta i}^{\gamma}\left(\mathbf{r}^{\prime}, \mathbf{r}\right) u_{\zeta i}^{\gamma}(\mathbf{r}), \\
\left(\hat{h}_{\zeta}^{\gamma}-\varepsilon_{\zeta i}^{\gamma}\right) G_{\zeta i}^{\gamma}\left(\mathbf{r}^{\prime}, \mathbf{r}\right)=\delta\left(\mathbf{r}-\mathbf{r}^{\prime}\right)-u_{\zeta i}^{\gamma}(\mathbf{r}) u_{\zeta i}^{\gamma *}\left(\mathbf{r}^{\prime}\right) .
\end{gathered}
$$

Using Eqs. (34)-(37) an integral equation for the effective exchange-correlation potential $V_{\zeta x c}^{\gamma}$ follows:

$$
\int H_{\zeta}^{\gamma}\left(\mathbf{r}, \mathbf{r}^{\prime}\right) V_{\zeta}^{\gamma}\left(\mathbf{r}^{\prime}\right) d \mathbf{r}^{\prime}=Q_{\zeta}^{\gamma}(\mathbf{r})
$$




$$
\begin{gathered}
H_{\zeta}^{\gamma}\left(\mathbf{r}, \mathbf{r}^{\prime}\right)=\sum_{i} u_{\zeta i}^{\gamma *}(\mathbf{r}) G_{\zeta i}^{\gamma}\left(\mathbf{r}, \mathbf{r}^{\prime}\right) u_{\zeta i}^{\gamma}\left(\mathbf{r}^{\prime}\right) \\
Q_{\zeta}^{\gamma}(\mathbf{r})=\sum_{i} \int d \mathbf{r}^{\prime} u_{\zeta i}^{\gamma *}(\mathbf{r}) G_{\zeta i}^{\gamma}\left(\mathbf{r}, \mathbf{r}^{\prime}\right) v_{\zeta x c, i}^{\gamma}\left(\mathbf{r}^{\prime}\right) u_{\zeta i}^{\gamma}\left(\mathbf{r}^{\prime}\right)
\end{gathered}
$$

The orbital dependent potential $v_{\zeta x c, i}^{\gamma}$ is given by

$$
v_{\zeta x c, i}^{\gamma}(\mathbf{r})=\frac{\delta E_{\zeta x c}^{\gamma}\left[u_{\zeta i}^{\gamma}\right]}{u_{\zeta i}^{\gamma} \delta u_{\zeta i}^{\gamma *}} .
$$

The effective exchange-correlation potential $V_{\zeta x c}^{\gamma}$ can be determined from the effective potential $V_{\zeta}^{\gamma}$ :

$$
V_{\zeta x c}^{\gamma}(\mathbf{r})=V_{\zeta}^{\gamma}-v-v_{J}^{\gamma}
$$

$v$ is the external potential. The classical Coulomb potential $v_{J}^{\gamma}$ slightly depends on $\gamma$ as the density $n^{\gamma}$ is somewhat different for the different multiplets.

To obtain the effective potential $V_{\zeta}^{\gamma}$ for the multiplet $\gamma$ the total energy $E^{\gamma}\left[u_{\zeta i}^{\gamma}\right]$ is needed. In the optimized potential method for nondegenerate states, the energy expression applied is the average of the total Hamiltonian $\hat{H}$ with a single Slater determinant (with the KohnSham orbitals, of course). Generalizing this idea, now the following energy expression is proposed for the multiplets:

$$
E^{\gamma}=\left\langle\tilde{\Psi}_{s i}^{\gamma}|\hat{H}| \tilde{\Psi}_{s i}^{\gamma}\right\rangle
$$

As it is well-known from the group theory, $E^{\gamma}$ is independent of $i$. Using Eq. (11) $E^{\gamma}$ is a functional of the one-electron orbitals $u_{\zeta i}^{\gamma}$ :

$$
E^{\gamma}=E^{\gamma}\left[u_{\zeta i}^{\gamma}\right]
$$

For the special case of atomic multiplets, it is a functional of the radial wave functions $P_{\zeta i}^{\gamma}$ :

$$
E^{\gamma}=E^{\gamma}\left[P_{\zeta i}^{\gamma}\right]
$$

It can also be written as: 


$$
E^{\gamma}=E_{a v}+\sum_{j} C_{j}^{\gamma} B_{j}^{\gamma}
$$

where $E_{a v}$ is the average energy of the different multiplets corresponding to a given configuration. The second term in the right hand side of Eq.(46) is responsible for the multiplet separation. The explicit form of Eq.(46) has to be given for the multiplet considered prior to the OPM calculation. Expressions for several atomic multiplets have already been derived. For example, for the $p^{2}$ electron configuration, the expression for $E^{\gamma}$ can be found in several textbooks (see e. g. [27]). They have the following form:

$$
\begin{aligned}
& E\left({ }^{3} P\right)=E_{a v}-\frac{3}{25} F^{2}(p p), \\
& E\left({ }^{1} D\right)=E_{a v}+\frac{3}{25} F^{2}(p p), \\
& E\left({ }^{1} S\right)=E_{a v}+\frac{12}{25} F^{2}(p p) .
\end{aligned}
$$

In this case there is only one term in the sum and $C_{1}^{\gamma}$ takes the value of $-3 / 25,3 / 25$ and $12 / 25$ for the multiplets ${ }^{3} P,{ }^{1} D$ and ${ }^{1} S$, respectively. $F^{2}(p p)$ is the Slater integral

$$
F^{2}(p p)=\iint R_{2 p}^{2}\left(r_{1}\right) R_{2 p}^{2}\left(r_{2}\right) \frac{r_{<}^{2}}{r_{>}^{3}} d r_{1} d r_{2}
$$

where $R_{2 p}$ is the radial wave function of the $2 p$ electrons. $r_{<}$means $r_{1}$ if it is smaller than $r_{2}$ and $r_{2}$ if it is smaller than $r_{1}$.

Now, in the knowledge of total energy expression, the OPM calculations can be performed solving Eqs. (38)-(41). One can also obtain the Hartree-Fock-like equations by taking the functional derivative of Eq. (46). For spherically symmetric systems we obtain the following radial equations:

$$
\left(-\frac{1}{2} \frac{d^{2}}{d r^{2}}+\frac{l_{i}\left(l_{i}+1\right)}{r^{2}}+v+v_{J}^{\gamma}+v_{\zeta x, i}^{\gamma}+w_{\zeta i}^{\gamma}\right) \tilde{P}_{\zeta i}^{\gamma}=\varepsilon_{\zeta i}^{\gamma} \tilde{P}_{\zeta i}^{\gamma},
$$

where $\tilde{P}=r \tilde{R}$. and

$$
w_{\zeta i}^{\gamma}=\sum_{j} C_{j}^{\gamma} \frac{1}{\tilde{P}_{\zeta i}^{\gamma}} \frac{d B_{j}^{\gamma}}{d \tilde{P}_{\zeta i}^{\gamma}}
$$


It is very difficult to calculate the effective potential $V_{\zeta}^{\gamma}$ because of vast numerical problems. Solution of the Hartree-Fock-like equations (51) is also very time-consuming. The KLI (Krieger, Li and Iafrate) [19] is an accurate approximate approach to OPM. Now we present a very simple, alternative derivation of the approximation based on a previous method of the present author [28].

After multiplying Eq. (51) by $\zeta$ and $P_{\zeta i}^{\gamma}$ and summing for all orbitals (from 1 to $M$ ) we obtain

$$
\zeta \sum_{i=1}^{M} \lambda_{i} \tilde{P}_{\zeta i}^{\gamma}\left(-\frac{1}{2} \frac{d^{2}}{d r^{2}}+\frac{l_{i}\left(l_{i}+1\right)}{r^{2}} \tilde{P}_{\zeta i}^{\gamma}\right)+\left(v+v_{J}^{\gamma}+v_{\zeta S}^{\gamma}+w_{\zeta}^{\gamma}\right) \varrho^{\gamma}=\zeta \sum_{i=1}^{M} \lambda_{i} \epsilon_{\zeta i}^{\gamma}\left(\tilde{P}_{\zeta i}^{\gamma}\right)^{2},
$$

where

$$
v_{\zeta S}^{\gamma}=\frac{\zeta}{\varrho^{\gamma}} \sum_{i=1}^{M} \tilde{P}_{\zeta i}^{\gamma} v_{\zeta x, i}^{\gamma} \tilde{P}_{\zeta i}^{\gamma}
$$

is the Slater potential and

$$
\varrho(\mathbf{r})^{\gamma}=\zeta \sum_{i}^{M} \lambda_{i}\left(\tilde{P}_{\zeta i}^{\gamma}(\mathbf{r})\right)^{2}
$$

is the density.

$$
w_{\zeta}^{\gamma}=\frac{\zeta}{\varrho^{\gamma}} \sum_{i=1}^{M} w_{\zeta i}^{\gamma}\left(\tilde{P}_{\zeta i}^{\gamma}\right)^{2}
$$

Then the modified Kohn-Sham equations (20) are multiplied by $\zeta$ and the orbitals $P_{\zeta i}^{\gamma}$ and summed for all orbitals

$$
\zeta \sum_{i=1}^{M} \lambda_{i} P_{\zeta i}^{\gamma}\left(-\frac{1}{2} \frac{d^{2}}{d r^{2}}+\frac{l_{i}\left(l_{i}+1\right)}{r^{2}} P_{\zeta i}^{\gamma}\right)+\varrho v_{\zeta K S}^{\gamma}=\zeta \sum_{i=1}^{M} \lambda_{i} \varepsilon_{\zeta i}^{\gamma}\left(P_{\zeta i}^{\gamma}\right)^{2}
$$

Now we consider the case when both equations (51) and (20) provide the same density $\varrho^{\gamma}$. Moreover, it is supposed that the orbitals $\tilde{P}_{\zeta i}^{\gamma}$ can be approximated by the orbitals $P_{\zeta i}^{\gamma}$. Then, comparing Eqs.(53) and (57) we obtain the generalized $\zeta$ KLI approximation for the Kohn-Sham potential

$$
v_{\zeta K S}^{\gamma}=v+v_{J}^{\gamma}+v_{\zeta x}^{\gamma}+w_{\zeta}^{\gamma},
$$


where $v_{\zeta x}^{\gamma}$ is the exchange potential and $w_{\zeta}^{\gamma}$ is the potential which is responsible for the multiplet separation.

$$
v_{\zeta x}^{\gamma}=v_{\zeta S}^{\gamma}+\frac{\zeta}{\varrho^{\gamma}} \sum_{i=1}^{M}\left\langle P_{\zeta i}^{\gamma}\left|\lambda_{i} v_{\zeta x}^{\gamma}-v_{\zeta x, i}^{\gamma}\right| P_{\zeta i}^{\gamma}\right\rangle\left|P_{\zeta i}^{\gamma}\right|^{2} .
$$

For $\zeta=1$ Eq. (59) gives the original KLI exchange potential for the multiplet. As we used Hartree-Fock like expression we obtained only the exchange. The results above are valid for any value of $\zeta$. We search that $\zeta_{c}$ for which the correlation energy disappears: $E_{\zeta_{c}, c}^{\gamma}[\varrho]=0$. For that value of $\zeta$ the $\zeta$ KLI method provides a very simple approximation that includes correlation.

\section{RESULTS AND DISCUSSION}

As an illustration the energies for the multiplets ${ }^{3} P,{ }^{1} D$ and ${ }^{1} S$ of the $\mathrm{C}, \mathrm{O}, \mathrm{S}$ and Si atoms are calculated using the $\zeta K L I$ method. The value $\zeta_{c}$ for which the $\zeta$ KLI and experimental energies [29] are equal has been determined. Table I presents these values $\zeta_{c}$. $\zeta>1$ means that the scaled number of electrons $N_{\zeta}$ is smaller than the true electron number $N$. The difference $q=N-N_{\zeta}$ is also shown in Table I. These values are about 0.04 in all cases.

The method presented here can be applied to molecules, too. To perform the calculation for a molecule one needs the explicit expression for the multiplet separation in Eq. (46). Therefore, one has to derive expressions similar to Eqs. (47) - (49). The $\zeta K L I$ method can be applied to molecules as well, though it is certainly more difficult perform KLI calculations for molecules.

The $\zeta K L I$ method is as simple as the original $K L I$ method. But it contains correlation as well. The $\zeta$ KLI method is not exact, because $E_{\zeta_{c}, c}^{\gamma}[\varrho]=0$ is valid only for a single density and the functional derivative, that is, the correlation potential is not zero. 
It can be easily shown that for a spherically symmetric case and equal weighting factors and supposing LS coupling, the Kohn-Sham equations have a very simple form In this case the noninteracting kinetic energy is given by

$$
T_{\zeta}^{\gamma}=\zeta \operatorname{tr}\left\{\hat{\Gamma}_{\zeta}^{\gamma} \hat{T}\right\}
$$

Substituting Eqs. (11) and (10) into Eq. (60) we obtain

$$
\begin{aligned}
T_{\zeta}^{\gamma}= & \zeta\left[(1-q) \sum_{i=1}^{g^{\gamma}} \omega_{i}^{\gamma} \sum_{\kappa} \sum_{\mu} c_{i, \kappa}^{\gamma *} c_{i, \mu}^{\gamma} \int \Phi_{\kappa}^{*}\left(-\sum_{j=1}^{N} \frac{1}{2} \nabla_{j}^{2}\right) \Phi_{\mu} d \mathbf{x}_{1}, \ldots, d \mathbf{x}_{N}\right. \\
& \left.+q \sum_{i=1}^{g^{i o n}} \omega_{i}^{i o n} \sum_{\kappa} \sum_{\mu} c_{i, \kappa}^{i o n *} c_{i, \mu}^{i o n} \int \Phi_{\kappa}^{*}\left(-\sum_{j=1}^{N-1} \frac{1}{2} \nabla_{j}^{2}\right) \Phi_{\mu} d \mathbf{x}_{1}, \ldots, d \mathbf{x}_{N-1}\right] .
\end{aligned}
$$

The Slater determinants $\Phi_{\kappa}$ are built of one-electron orbitals

$$
u_{j}^{\gamma}=P_{n l}^{\gamma} Y_{l m_{l}} \sigma_{m_{s}},
$$

corresponding to the given electron configuration. $P_{n l}^{\gamma}$ are radial wave functions with quantum numbers $n$ and $l, Y_{l m_{l}}$ are the spherical harmonics and $\sigma_{m_{s}}$ stands for the spin eigenfunction. The Slater determinants $\Phi_{\kappa}$ and $\Phi_{\mu}$ may differ only in orbitals of the open shells, that is in the quantum numbers $m_{l}$ and $m_{s}$. Taking into consideration that the spherical harmonics are eigenfunctions of the Laplatian and the orthogonality of the wave functions in Eq. (62) we notice that the integrals taken with different determinants disappear in Eq. (61). So the noninteracting kinetic energy is given by

$$
\begin{aligned}
T_{\zeta}^{\gamma}= & \zeta\left[(1-q) \sum_{i=1}^{g^{\gamma}} \omega_{i}^{\gamma} \sum_{\kappa}\left|c_{i, \kappa}^{\gamma}\right|^{2} \sum_{j=1}^{N} \lambda_{\kappa, j}^{\gamma}\left\langle u_{j}\left|-\frac{1}{2} \nabla^{2}\right| u_{j}\right\rangle\right. \\
& \left.+q \sum_{i=1}^{g^{i o n}} \omega_{i}^{i o n} \sum_{\kappa}\left|c_{i, \kappa}^{i o n}\right|^{2} \sum_{j=1}^{N-1} \lambda_{\kappa, j}^{i o n}\left\langle u_{j}\left|-\frac{1}{2} \nabla^{2}\right| u_{j}\right\rangle\right] .
\end{aligned}
$$

Now, the occupation numbers $\lambda_{\kappa, j}^{\gamma}$ do not depend on $\kappa$ because we have the same electron configuration. (e.g. in the case studied in Section IV. we have $p^{2}$, i.e. the occupation numbers are 2 for the p electrons in each determinant.) Making use of the orthogonality of the wave functions $\Psi_{s, i}^{\gamma}$ and the equality of the weighting factors $\omega_{M i}^{\gamma}$ we arrive at Eq. (18). 
Eq. (19) can be similarly derived. The density is given by

$$
\begin{aligned}
n_{\zeta}^{\gamma}= & {\left[(1-q) \sum_{i=1}^{g^{\gamma}} \omega_{i}^{\gamma} \sum_{\kappa} \sum_{\mu} c_{i, \kappa}^{\gamma *} c_{i, \mu}^{\gamma} \int \Phi_{\kappa}^{*} \Phi_{\mu} d s_{1} d \mathbf{x}_{2}, \ldots, d \mathbf{x}_{N}\right.} \\
& \left.+q \sum_{i=1}^{g^{i o n}} \omega_{i}^{i o n} \sum_{\kappa} \sum_{\mu} c_{i, \kappa}^{i o n *} c_{i, \mu}^{i o n} \int \Phi_{\kappa}^{*} \Phi_{\mu} d s_{1} d \mathbf{x}_{2}, \ldots, d \mathbf{x}_{N-1}\right] .
\end{aligned}
$$

Taking into account that $\Phi_{\kappa}$ and $\Phi_{\mu}$ should correspond to the same quantum numbers $M_{L}$ and $M_{S}$ and the orthogonality of the wave functions in Eq. (62) we arrive at Eq. (19).

\section{REFERENCES}

[1] P. Hohenberg and W. Kohn, Phys. Rev. B 136, 864 (1964).

[2] B. I. Dunlap, Adv. Chem. Phys. 69, 287 (1987).

[3] R. McWeeney, Phil. Mag. B 69, 727 (1994).

[4] J. P. Perdew, A. Savin and K. Burke, Phys. Rev. A 51,4531(1995).

[5] A. Görling, Phys. Rev. A 47, 2783 (1993).

[6] P.S. Bagus and B.I. Bennett, Int. J. Quantum Chem. 9, 143 (1975).

[7] T. Ziegler, A. Rauk and E.J. Baerends, Theor. Chim. Acta (Berl)43, 261 (1977).

[8] U. von Barth, Phys. Rev. A 20, 1693 (1979).

[9] J. H. Wood, J. Phys. B 13, 1 (1980).

[10] E.K.U. Gross, L.N. Oliveira and W. Kohn, Phys. Rev. A 37, 2805, 2809, 2821, (1988).

[11] Á. Nagy, Int. J. Quantum. Chem.S. 29, 297 (1995).

[12] A.K. Theophilou, J. Phys. C 12, 5419 (1978);

[13] Á. Nagy, Phys. Rev. A 57, 1672 (1998).

[14] Á. Nagy, J. Phys. B 32, 2841 (1999).

[15] G. K.-L. Chan and N. C. Handy, Phys. Rev. A 59, 2670 (1999).

[16] Á. Nagy, Chem. Phys. Lett. 411492 (2005). 
[17] Á. Nagy, J. Chem. Phys. 123044105 (2005).

[18] R. T. Sharp and G. K. Horton, Phys. Rev. A 30,317(1953); K. Aashamar, T. M. Luke and J. D. Talman, At. Data Nucl. Data Tables 22443 (1978).

[19] J. B. Krieger, Y. Li and G. J. Iafrate, Phys. Lett. A 146,256(1990) ; Phys. Rev. A 45,101(1992); Phys. Rev. A 46, 5453(1992); Int. J. Quantum. Chem. 41,489(1992).

[20] M. Levy, Proc. Natl. Acad. Sci. USA 76, 6062 (1979).

[21] E. H. Lieb, Int. J. Quantum. Chem. 24, 243 (1983).

[22] W. Kohn, L.J. Sham, Phys. Rev.140 A, 1133 (1965).

[23] Parr R. G.and Yang W., Density Functional Theory of Atoms and Molecules (Oxford Univ. Press, New York, 1989).

[24] Further references in Nagy Á, 1998, Phys. Rep. 3981.

[25] Dreizler R. M. and Gross E.K.U. , 1990, Density Functional Theory (Springer-Verlag: Berlin).

[26] B. Weiner and S. Trickey, discussion at the Sanibel Symposium, 1985.

[27] J. C. Slater, Quantum Theory of Atomic Structure ( New York, McGraw-Hill,1960 ) vol. 1.

[28] Á. Nagy, Phys. Rev. A 553465 (1997).

[29] C. E. Moore, Atomic Energy Levels NBS Circular No 467. (Washington, DC. US Govt. Printing Office, 1949).

\section{ACKNOWLEDGEMENTS}

This work is supported by TAMOP 4.2.1-08/1-2008-003 project. The project is implemented through the New Hungary Development Plan co-financed by the European Social Fund, and the European Regional Development Fund. Grant OTKA No. K 67923 is also gratefully acknowledged. 
TABLE 1

Hartree-Fock, $\zeta$ KLI and experimental total energies [29] (in Ry) for the multiplets ${ }^{3} P,{ }^{1} D$ and ${ }^{1} S$ of the C, O, S and Si atoms. The values of $\zeta$ and $q$ for which the $\zeta$ KLI and experimental total energies are equal.

\begin{tabular}{|l|l|c|c|c|c|}
\hline & & $\mathrm{C}$ & $\mathrm{O}$ & $\mathrm{Si}$ & $\mathrm{S}$ \\
\hline \hline${ }^{3} P$ & $H F$ & -75.3772 & -149.6187 & -577.7085 & -795.0095 \\
& $K L I$ & -75.3724 & -149.6147 & -577.6975 & -794.9976 \\
& $E x p$ & -75.6904 & -150.1346 & -578.7500 & -796.2780 \\
& $\zeta$ & 1.00661 & 1.00661 & 1.00300 & 1.00265 \\
\hline${ }^{1} D$ & $H F$ & -75.2626 & -149.4584 & -577.6300 & -794.9042 \\
& $K L I$ & -75.2583 & -149.4543 & -577.6176 & -794.8909 \\
& Exp & -75.5979 & -149.9900 & -578.6926 & -796.1938 \\
& $\zeta$ & 1.00715 & 1.00608 & 1.00308 & 1.00270 \\
& $q$ & 0.04257 & 0.04831 & 0.04300 & 0.04308 \\
\hline${ }^{1} S$ & $H F$ & -75.0991 & -149.2219 & -577.5169 & -794.7489 \\
& $K L I$ & -75.0850 & -149.2141 & -577.4797 & -794.7224 \\
& Exp & -75.4931 & -149.8267 & -578.6097 & -796.0759 \\
\hline & 1.00874 & 1.00701 & 1.00326 & 1.00281 \\
\hline & 0.05201 & 0.05567 & 0.04546 & 0.04491 \\
\hline
\end{tabular}

